\title{
Radiological and Surgical Implications of Neoadjuvant Treatment With FOLFIRINOX for Locally Advanced and Borderline Resectable Pancreatic Cancer
}

\author{
Cristina R. Ferrone, MD*, Giovanni Marchegiani, $\mathbf{M D}^{\star}$, Theodore S. Hong, $\mathbf{M D}^{\dagger}$, David $\mathbf{P}$. \\ Ryan, MD $\ddagger$, Vikram Deshpande, MD $\ddagger$, Erin I. McDonnell ${ }^{\ddagger}$, Francesco Sabbatino, PhD $^{\star}$, \\ Daniela Dias Santos, MD*, Jill N. Allen, MD ${ }^{\ddagger}$, Lawrence S. Blaszkowsky, MD ${ }^{\ddagger}$, Jeffrey W. \\ Clark, MD $\ddagger$, Jason E. Faris, MD $\ddagger$, Lipika Goyal, MD $\ddagger$, Eunice L. Kwak, PhD ${ }^{\ddagger}$, Janet E. \\ Murphy, MD $\ddagger$, David T. Ting, MD $\ddagger$, Jennifer Y. Wo, MD $\dagger$, Andrew X. Zhu, PhD ${ }^{\ddagger}$, Andrew L. \\ Warshaw, MD*, Keith D. Lillemoe, MD*, and Carlos Fernández-del Castillo, MD* \\ *Department of Surgery, Massachusetts General Hospital, Harvard Medical School, Boston, MA \\ †Department of Radiation Oncology, Massachusetts General Hospital, Harvard Medical School, \\ Boston, MA \\ ¥Department of Medical Oncology, Massachusetts General Hospital, Harvard Medical School, \\ Boston, MA
}

\begin{abstract}
Purpose-On the basis of the ACCORD trial, FOLFIRINOX is effective in metastatic pancreatic adenocarcinoma (PDAC), making it a rational choice for locally advanced PDAC (LA). Aims of this study are to evaluate the accuracy of imaging in determining the resectability of PDAC and to determine the surgical and clinicopathologic outcomes of pancreatic resections after neoadjuvant FOLFIRINOX therapy.
\end{abstract}

Patients and Methods-Clinicopathologic data were retrospectively collected for surgical PDAC patients receiving neoadjuvant FOLFIRINOX or no neoadjuvant therapy between April 2011 and February 2014. Americas Hepato-Pancreato-Biliary Association/Society of Surgical Oncology/Society for Surgery of the Alimentary Tract consensus guidelines defined LA and borderline. Imaging was reviewed by a blinded senior pancreatic surgeon.

Results-Of 188 patients undergoing resection for PDAC, 40 LA/borderline received FOLFIRINOX and 87 received no neoadjuvant therapy. FOLFIRINOX resulted in a significant decrease in tumor size, yet 19 patients were still classified as LA and 9 as borderline. Despite postFOLFIRINOX imaging suggesting continued unresectability, 92\% had an R0 resection. When compared with no neoadjuvant therapy, FOLFIRINOX resulted in significantly longer operative times (393 vs 300 minutes) and blood loss (600 vs $400 \mathrm{~mL}$ ), but significantly lower operative

\footnotetext{
Copyright $(92014$ by Lippincott Williams \& Wilkins

Reprints: Cristina R. Ferrone, MD, Department of Surgery, Harvard Medical School, Massachusetts General Hospital, WANG 460, 15 Parkman Street, Boston, MA 02108, cferrone @ partners.org.

Disclosure: The authors declare no conflicts of interest.

Presented at the SSO 67th Cancer Symposium in Phoenix, AZ.
} 
morbidity (36\% vs 63\%) and no postoperative pancreatic fistulas. Length of stay (6 vs 7 days), readmissions ( $20 \%$ vs $30 \%$ ), and mortality were equivalent ( $1 \%$ vs $0 \%$ ). On final pathology, the FOLFIRINOX group had a significant decrease in lymph node positivity (35\% vs 79\%) and perineural invasion (72\% vs 95\%). Median follow-up was 11 months with a significant increase in overall survival with FOLFIRINOX.

Conclusions-After neoadjuvant FOLFIRINOX imaging no longer predicts unresectability. Traditional pathologic predictors of survival are improved, and morbidity is decreased in comparison to patients with clearly resectable cancers at the time of presentation.

\section{Keywords}

chemotherapy; downstaging; FOLFIRINOX; neoadjuvant therapy; pancreas cancer; pancreatic adenocarcinoma; PDAC; surgery

The prevalence of pancreatic adenocarcinoma (PDAC) continues to increase. The American Cancer Society predicts 46,420 new cases and 39,590 deaths in the United States in 2014. The majority of patients present with metastatic disease, whereas $30 \%$ present with locally advanced (LA) cancers. ${ }^{1}$ Unfortunately, overall survival (OS) of pancreatic cancer patients remains low, with surgical resection offering the only chance for potential cure. However, even then the OS is reported to be less than $20 \% .^{2-4}$ In the hopes of rendering LA PDAC patients resectable, combination chemotherapy often followed by 50.4 Gy of radiation with low-dose chemotherapy is administered. 5,6

However, historically only $19 \%$ to $30 \%$ of patients are rendered resectable. ${ }^{7-10}$ If an R0 resection can be achieved, OS is similar to patients who are considered resectable at presentation. ${ }^{9}$ Unfortunately, these heavily treated patients with LA PDAC have an increased surgical morbidity and mortality. ${ }^{9,11}$

A significant breakthrough for patients with metastatic PDAC was achieved in 2011 with the ACCORD trial. ${ }^{12}$ This trial demonstrated that the OS with fluorouracil, leucovorin, oxaliplatin, and irinotecan (FOLFIRINOX) was improved when compared with gemcitabine for patients with metastatic pancreatic cancer ( 6.8 vs 11.1 months, $P<0.001) .{ }^{12}$ On the basis of these encouraging results, FOLFIRINOX became a rational choice to render patients with LA PDAC resectable. ${ }^{13}$

Resectability of PDAC is determined by radiologic imaging. The most commonly used imaging modality to stage PDAC is a triple-phase contrast-enhanced thin-slice (multidetector row) helical computed tomography with 3-dimensional reconstructions. Unfortunately, the definitions for LA and borderline lesions vary significantly. This ambiguity prompted 4 guideline statements from the National Comprehensive Cancer Network, the University of Texas MD Anderson Cancer Center, the Americas HepatoPancreato-Biliary Association (AHPBA)/Society of Surgical Oncology (SSO)/Society for Surgery of the Alimentary Tract (SSAT). ${ }^{14-17}$ These guidelines address vascular involvement, which is a major determinant of unresectability.

This study provides the largest series of neoadjuvantly treated FOLFIRINOX patients who underwent surgical resection. Radiologic and clinicopathologic features of patients with 
either LA or borderline resectable pancreatic cancer who received neoadjuvant FOLFIRINOX were compared with resectable patients who underwent exploration without neoadjuvant treatment. The first aim of this study is to critically evaluate the accuracy of imaging in determining resectability after FOLFIRINOX with or without chemoradiation. The second aim is to compare the surgical outcomes and clinicopathologic results of pancreatic resections in this cohort of FOLFIRINOX-treated patients with patients who received no neoadjuvant therapy.

\section{Methods}

With approval from the institutional review board, retrospective and prospectively collected clinicopathologic data were collected for all patients who underwent resection for PDAC between April 2011 and March 1, 2014, and for all patients who were surgically explored after neoadjuvant treatment with FOLFIRINOX. Clinicopathologic factors evaluated included age at operation, sex, race (grouped as white, Hispanic, and other), the Charlson comorbidity index, ${ }^{18}$ body mass index (BMI) coded as underweight $\left(<18.5 \mathrm{~kg} / \mathrm{m}^{2}\right)$, normal $\left(18.5-24.9 \mathrm{~kg} / \mathrm{m}^{2}\right)$, obese $\left(25-35 \mathrm{~kg} / \mathrm{m}^{2}\right)$, and morbidly obese $\left(>35 \mathrm{~kg} / \mathrm{m}^{2}\right)$ and previous history of cancer. Baseline CA 19-9 level (U/mL), size and vascular involvement on preoperative CT, estimated intraoperative blood loss, type of surgery (Whipple procedure, distal or total pancreatectomy), hospital length of stay, postoperative complications according to Clavien-Dindo classification, ${ }^{19}$ intraoperative radiation therapy, and postoperative chemotherapy. Disease-free survival and OS were calculated from the date of diagnosis. Date of death was obtained either from the medical records or from the Social Security Death Index.

\section{Histologic Characteristics}

Pathologic data collected included tumor grade (classified as well, moderately, poorly differentiated, and undifferentiated), tumor size, TNM classification, number of positive nodes, resection margins, perineural invasion, lymphovascular invasion, and American Joint Committee on Cancer stage.

A positive microscopic resection margin was defined as presence of tumor cells on any surgical specimen margin as described by Staley et al. ${ }^{20}$ We recorded the pancreatic transection, posterior retroperitoneal, uncinate, common bile duct, and enteric margins. A gastrointestinal pathologist specialized in pancreatic cancer confirmed the histologic diagnosis.

\section{Radiologic Characteristics}

Triple-phase contrast-enhanced thin-slice (multidetector row) helical CT with 3-dimensional reconstruction and magnetic resonance images were reviewed. All patients were reviewed at our gastrointestinal multidisciplinary meeting. This tumor board includes 2 or more pancreatic surgeons, 1 or more gastrointestinal radiologist, 2 or more medical oncologists, and 2 or more gastrointestinal radiation oncologists. LA and borderline lesions were defined on the basis of the AHPBA/SSO/SSAT consensus guidelines. ${ }^{17}$ No patient had evidence of metastases or distant nodal disease. Patients were considered LA if there was long segment 
occlusion of the mesenteric vein/portal vein, more than 180-degree involvement of the superior mesenteric artery (SMA) or involvement of the hepatic artery or celiac trunk. Borderline resectable lesions included lesions with tumor abutment and short segment occlusion of the mesenteric vein/portal vein, gastroduodenal artery encasement up to the hepatic artery without extension to the celiac axis, or $<180$ degrees of tumor abutment of the SMA.

A retrospective blinded review of the radiology before and after treatment with FOLFIRINOX was performed by a senior pancreatic surgeon (A.L.W.).

\section{Statistical Analysis}

Statistical analyses were performed with SAS software version 9.3 (Copyright 2010, SAS Institute Inc, Cary, NC). Continuous data were expressed as median and range, whereas categorical data were expressed as frequencies and percentages. Across neoadjuvant therapy groups, the T test and Wilcoxon rank sum tests were used to compare normally and nonnormally distributed continuous data, respectively, whereas the Fisher exact test was used to compare categorical data. Comparisons over time within a neoadjuvant therapy group were made with a paired $\mathrm{T}$ test for continuous data and the McNemar test for categorical data. For OS, the Kaplan-Meier method was used. The difference in survival was tested with log-rank test.

\section{Results}

\section{Overall Cohort}

A total of 188 patients underwent pancreatic resection for PDAC. Of these 188 patients, 40 received neoadjuvant FOLFIRINOX with or without chemoradiation for LA or borderline resectable PDAC, and 87 received no neoadjuvant therapy (Fig. 1). The patients who received no neoadjuvant therapy were determined to have resectable cancers on preoperative imaging and declined participation or did not qualify for other neoadjuvant protocols. These 2 groups are the basis of this report.

This study compares the best and worst "actors" by comparing patients with LA or borderline cancers who received FOLFIRINOX with those patients who received no neoadjuvant therapy because of the presence of what was believed to be a clearly resectable lesion. The clinicopathologic factors of the study groups are listed in Table 1.

LA and borderline patients who received neoadjuvant FOLFIRINOX were younger and had a lower Charlson comorbidity score and better ECOG (Eastern Cooperative Oncology Group) performance status when compared with the resectable patients who received no neoadjuvant therapy. Patients receiving FOLFIRINOX also had a significantly lower BMI.

\section{FOLFIRINOX Patients}

A total of 47 patients underwent neoadjuvant treatment with FOLFIRINOX followed by surgical exploration for attempted resection. The patients received a median of 8 complete cycles (range 1-24), and only 3 patients (6.3\%) developed severe treatment-related toxicity resulting in disruption of therapy. Chemoradiation with 50.4 Gy and 5-FU (fluorouracil) was 
administered to 24 patients who had no evidence of progressive disease after FOLFIRINOX and before surgical exploration.

Patients receiving neoadjuvant FOLFIRINOX had a decrease in pre- and posttreatment median Ca19-9 from 169 to 16 . This resulted in a Ca19-9 less than $40 \mathrm{U}$ in $72 \%$ of patients compared with only $31 \%$ of patients pretreatment. A significant decrease in tumor diameter on CT from a median of $3.6 \mathrm{~cm}$ to $2.2 \mathrm{~cm}$ was also observed.

Of the 47 patients, 3 had metastatic disease and 4 were LA and unresectable. A pancreaticoduodenectomy was performed in 34 patients and a distal pancreatectomy in 6 patients. Intraoperative radiation therapy administering a 7 to $12 \mathrm{~Gy}$ boost to the tumor bed was performed in 12 patients who underwent resection of their PDAC.

\section{Pre- and Post-FOLFIRINOX Staging}

On the basis of the AHPBA/SSO/SSAT consensus guidelines, 25 patients were considered LA and 15 were considered borderline at the time of diagnosis by the multidisciplinary tumor board (see Table 2). Posttreatment review of the imaging by the tumor board identified a complete response in 6 patients, a partial response in 30 patients, and stable disease in 4 patients. No progression of disease was identified.

To obtain an objective assessment of preoperative tumor staging, all pre- and postFOLFIRINOX imaging was reviewed by a senior pancreatic surgeon (A.L.W.) who was blinded to the patient's clinical history and the timing (pre- or post-FOLFIRINOX) of the imaging. This individual did not participate in the care of any of these patients. After review of the pretreatment imaging, 26 cancer patients were classified as LA and 14 as borderline. After neoadjuvant treatment, 19 were classified as LA, 9 as borderline, and 12 as resectable (Figs. 2A, B).

\section{Operative Outcomes}

Operative outcomes for the resectable patients were compared with the 40 resectable patients who received FOLFIRINOX neoadjuvantly (Table 3). Pancreaticoduodenectomy was the most common operation. Median operative time was significantly longer in the FOLFIRINOX patients, when compared with the resectable group not receiving neoadjuvant therapy. This increase in operative time was most likely due to the additional dissection of critical vessels such as the SMA. Five venous resections were performed. These more technically challenging operations resulted in a significant increase in blood loss. Despite the longer operation and increased blood loss, overall postoperative morbidity was decreased in the FOLFIRINOX group. Specifically, no patients in the FOLFIRINOX group developed a pancreatic fistula. In addition, length of stay, mortality, and read-missions were equivalent to those of the upfront resectable patients (Table 3).

\section{Pathologic Results}

Final pathology of the resected cancer demonstrated significant downstaging of the LA or borderline cancer in patients treated with neoadjuvant FOLFIRINOX with or without chemoradiation. Only $35 \%$ of patients had positive lymph nodes, compared with $79 \%$ of 
patients who went directly to the operating room $(<0.001)$ (Table 4). Patients treated with FOLFIRINOX also had significantly smaller tumors and lower rates of lymphovascular and perineural invasion (Table 4). An R0 resection was achieved in 92\% of patients, compared with $86 \%$ of patients receiving no neoadjuvant therapy $(P=\mathrm{NS})$. Four patients treated with FOLFIRINOX had minimal $(<1 \mathrm{~mm})$ cancer and 2 no evidence of cancer on pathologic examination, consistent with a complete response.

\section{Disease-Free and Overall Survival}

Median follow-up for the entire cohort was 11 months. For the patients receiving neoadjuvant FOLFIRINOX median follow-up was 13 months. Progression was documented in $38 \%$ of patients receiving neoadjuvant FOLFIRINOX, compared with $49 \%$ of patients receiving no neoadjuvant therapy. Distant disease was the most common first site of progression for both cohorts.

Patterns of progression are listed in Table 3. Median OS for the entire cohort was 34 months. Patients who received FOLFIRINOX and underwent surgical resection had a significant increase in OS compared with the group of patients with clearly resectable tumors who received no neoadjuvant therapy $(P=0.008)($ Fig. 3$)$. Both patients who had a complete response are alive and free of disease at 8 months and 16 months.

\section{Discussion}

The majority of patients with PDAC present with metastatic or LA tumors. Historically neoadjuvant chemoradiation therapy rendered approximately one third of LA cancers resectable. ${ }^{9,21}$ These patients, despite resection, recurred quickly with a median survival of 14 to 22 months. ${ }^{9,21}$ In the era of more effective chemotherapy with FOLFIRINOX, there has been a significant improvement in the median survival of patients with metastatic disease, suggesting that this more aggressive approach may be useful in patients with LA and borderline PDAC.

This study demonstrates that the historical criteria for determining resectability by crosssectional imaging are no longer appropriate in patients who receive FOLFIRINOX with or without radiation therapy. This is the likely basis for the apparent persistence of criteria for unresectability on imaging. A senior pancreatic surgeon blinded to the timing of the scans deemed the majority of patients as still borderline or LA after therapy; however, the R0 resection rate was 92\%. Despite a decrease in Ca19-9 levels and/or a decrease in the tumor size, clear fat planes around critical vascular structures were not present on post-

FOLFIRINOX preoperative imaging. Pathologic analysis demonstrated no viable cancer, but significant fibrosis from the therapy. Current radiologic imaging cannot distinguish between fibrosis and viable cancer, which is the basis for the continued criteria for unresectability on imaging. Our series suggests that despite the lack of historically accepted criteria for resection on imaging, patients should be explored for attempted resection. Involved arterial structures or narrowing of venous structures should be approached via serial frozen-section biopsies before attempted resection. If biopsies are positive, resection should be abandoned because an $\mathrm{R} 1$ or $\mathrm{R} 2$ resection is associated with a poor OS. ${ }^{22,23}$ Appropriate palliative measures should be considered. 
Patients who received FOLFIRINOX in this study were deemed either unresectable or borderline. The FOLFIRINOX patients presented with more advanced cancers but were younger and had a better ECOG performance score than patients who received an upfront operation. However, despite the advanced nature of the cancers, the aggressive neoadjuvant therapy, the prolonged operations, and increased intraoperative blood loss, patients treated with FOLFIRINOX had a decreased postoperative morbidity compared with patients presenting with clearly resectable disease. It is critical to note that no patients treated with preoperative FOLFIRINOX experienced a pancreatic fistula, a major complication of pancreatic cancer resections. A zero pancreatic fistula rate was also observed by Christians et al. ${ }^{24}$ The technically more challenging operations in the FOLFIRINOX patients also did not lead to increased mortality, length of stay, or 90-day readmission rates.

Imaging was unable to predict the encouraging pathologic results obtained in the patients who received FOLFIRINOX with or without chemoradiation. A significant decrease in lymphovascular and perineural invasion, as well as lymph node positivity, was identified after FOLFIRINOX. Pathologic evidence of minimal to no tumor was demonstrated in 15\% of cases. Similar encouraging pathologic responses were described in the 12 borderline patients resected by Christians et al. ${ }^{24}$ If traditional prognostic markers continue to be valid, improvements in node positivity, lymphovascular invasion, perineural invasion, and R0 resection should translate into improved survival.

On the basis of early short-term follow-up, patients who presented with LA or borderline resectable PDAC and received neoadjuvant FOLFIRINOX with or without chemoradiation had an improved OS when compared with patients who presented with resectable disease and received no neoadjuvant therapy. A longer follow-up and more patients will be necessary to confirm these results.

\section{Conclusions}

In summary, FOLFIRINOX with or without chemoradiation therapy has demonstrated impressive improvements in patients with LA PDAC. Traditional imaging criteria for resectability seem no longer to be accurate when evaluating posttreatment imaging. A more aggressive surgical approach, which involves serial biopsies around involved vascular structures before attempted resection, is justified by these findings. Because of the novelty of this regimen, OS and disease-free survival data are still early, but the results are encouraging. The improvement of traditional prognostic markers of survival and our early survival data may translate into an increased long-term OS.

\section{References}

1. Hidalgo M. Pancreatic cancer. New Engl J Med. 2010; 362:1605-1617. [PubMed: 20427809]

2. Ferrone CR, Pieretti-Vanmarcke R, Bloom JP, et al. Pancreatic ductal adenocarcinoma: long-term survival does not equal cure. Surgery. 2012; 152:S43-S49. [PubMed: 22763261]

3. Ferrone CR, Brennan MF, Gonen M, et al. Pancreatic adenocarcinoma: the actual 5-year survivors. J Gastrointest Surg. 2008; 12:701-706. [PubMed: 18027062]

4. Winter JM, Cameron JL, Campbell KA, et al. 1423 pancreaticoduodenectomies for pancreatic cancer: a single-institution experience. J Gastrointest Surg. 2006; 10:1199-1210. discussion 12101211. [PubMed: 17114007] 
5. Ko AH, Quivey JM, Venook AP, et al. A phase II study of fixed-dose rate gemcitabine plus lowdose cisplatin followed by consolidative chemoradiation for locally advanced pancreatic cancer. Int J Radiat Oncol Biol Phys. 2007; 68:809-816. [PubMed: 17363191]

6. Ben-Josef E, Shields AF, Vaishampayan U, et al. Intensity-modulated radiotherapy (IMRT) and concurrent capecitabine for pancreatic cancer. Int J Radiat Oncol Biol Phys. 2004; 59:454-959. [PubMed: 15145162]

7. White RR, Hurwitz HI, Morse MA, et al. Neoadjuvant chemoradiation for localized adenocarcinoma of the pancreas. Ann Surg Oncol. 2001; 8:758-765. [PubMed: 11776488]

8. Landry J, Catalano PJ, Staley C, et al. Randomized phase II study of gemcitabine plus radiotherapy versus gemcitabine, 5-fluorouracil, and cisplatin followed by radiotherapy and 5-fluorouracil for patients with locally advanced, potentially resectable pancreatic adenocarcinoma. J Sur Oncol. 2010; 101:587-592.

9. Gillen S, Schuster T, Meyer Zum Buschenfelde C, et al. Preoperative/neoadjuvant therapy in pancreatic cancer: a systematic review and meta-analysis of response and resection percentages. PLoS Med. 2010; 7:e1000267. [PubMed: 20422030]

10. Cai S, Hong TS, Goldberg SI, et al. Updated long-term outcomes and prognostic factors for patients with unresectable locally advanced pancreatic cancer treated with intraoperative radiotherapy at the Massachusetts General Hospital, 1978 to 2010. Cancer. 2013; 119:4196-4204. [PubMed: 24006012]

11. He J, Page AJ, Weiss M, et al. Management of borderline and locally advanced pancreatic cancer: where do we stand? World J Gastroenterol. 2014; 20:2255-2266. [PubMed: 24605025]

12. Conroy T, Desseigne F, Ychou M, et al. FOLFIRINOX versus gemcitabine for metastatic pancreatic cancer. New Engl J Med. 2011; 364:1817-1825. [PubMed: 21561347]

13. Faris JE, Blaszkowsky LS, McDermott S, et al. FOLFIRINOX in locally advanced pancreatic cancer: the Massachusetts General Hospital Cancer Center experience. Oncologist. 2013; 18:543548. [PubMed: 23657686]

14. Katz MH, Pisters PW, Evans DB, et al. Borderline resectable pancreatic cancer: the importance of this emerging stage of disease. J Am Coll Surg. 2008; 206:833-846. discussion 846-848. [PubMed: 18471707]

15. Katz MH, Marsh R, Herman JM, et al. Borderline resectable pancreatic cancer: need for standardization and methods for optimal clinical trial design. Ann Surg Oncol. 2013; 20:27872795. [PubMed: 23435609]

16. Tempero MA, Arnoletti JP, Behrman SW, et al. Pancreatic Adenocarcinoma, version 2.2012: featured updates to the NCCN Guidelines. J Natl Compr Canc Netw. 2012; 10:703-713. [PubMed: 22679115]

17. Callery MP, Chang KJ, Fishman EK, et al. Pretreatment assessment of resectable and borderline resectable pancreatic cancer: expert consensus statement. Ann Surg Oncol. 2009; 16:1727-1733. [PubMed: 19396496]

18. Charlson ME, Pompei P, Ales KL, et al. A new method of classifying prognostic comorbidity in longitudinal studies: development and validation. J Chronic Dis. 1987; 40:373-383. [PubMed: 3558716]

19. Dindo D, Demartines N, Clavien PA. Classification of surgical complications: a new proposal with evaluation in a cohort of 6336 patients and results of a survey. Ann Surg. 2004; 240:205-213. [PubMed: 15273542]

20. Staley CA, Cleary KR, Abbruzzese JL, et al. The need for standardized pathologic staging of pancreaticoduodenectomy specimens. Pancreas. 1996; 12:373-380. [PubMed: 8740405]

21. Andriulli A, Festa V, Botteri E, et al. Neoadjuvant/preoperative gemcitabine for patients with localized pancreatic cancer: a meta-analysis of prospective studies. Ann Surg Oncol. 2012; 19:1644-1662. [PubMed: 22012027]

22. Konstantinidis IT, Warshaw AL, Allen JN, et al. Pancreatic ductal adenocarcinoma: is there a survival difference for $\mathrm{R} 1$ resections versus locally advanced unresectable tumors? What is a "true" R0 resection? Ann Surg. 2013; 257:731-736. [PubMed: 22968073] 
23. Howard TJ, Krug JE, Yu J, et al. A margin-negative R0 resection accomplished with minimal postoperative complications is the surgeon's contribution to long-term survival in pancreatic cancer. J Gastrointest Surg. 2006; 10:1338-1345. discussion 1345-1346. [PubMed: 17175452]

24. Christians KK, Tsai S, Mahmoud A, et al. Neoadjuvant FOLFIRINOX for borderline resectable pancreas cancer: a new treatment paradigm? Oncologist. 2014; 19:266-274. [PubMed: 24569947] 


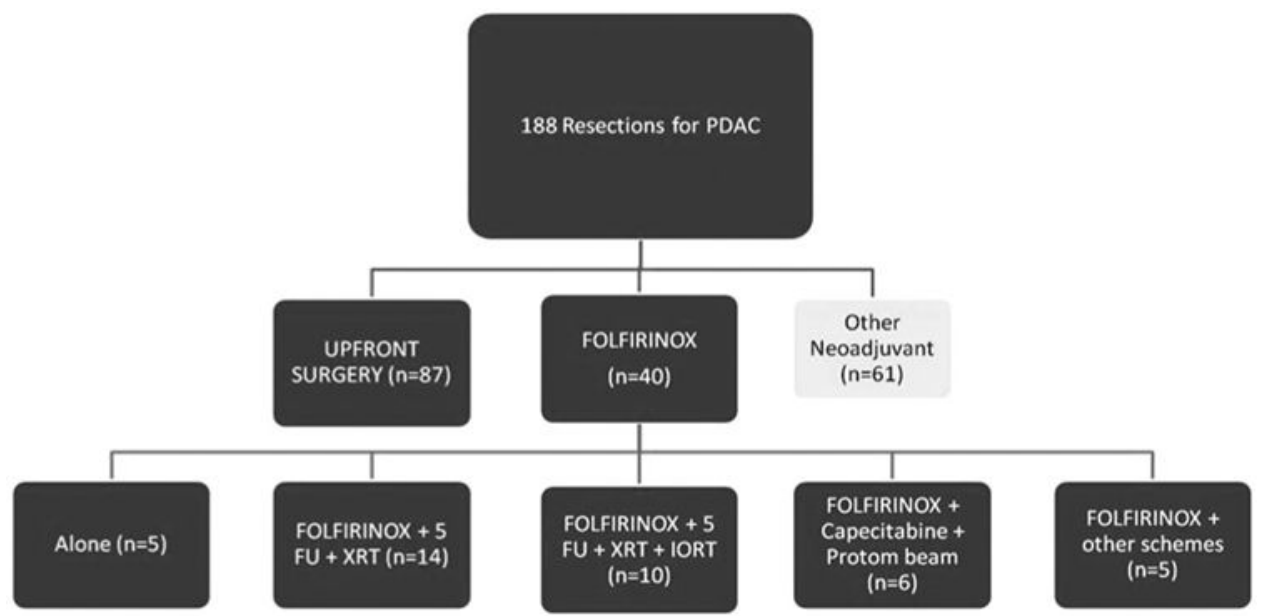

Figure 1.

Clinical treatment flow-chart. 


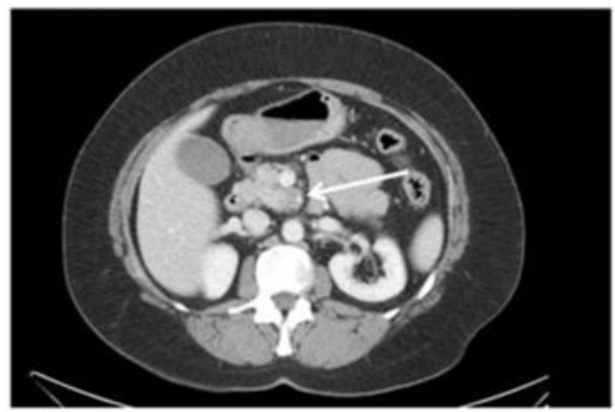

Pre FOLFIRINOX

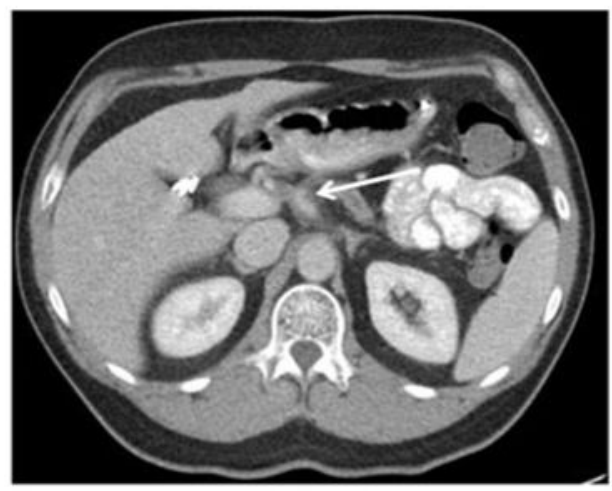

Celiac trunk involvement

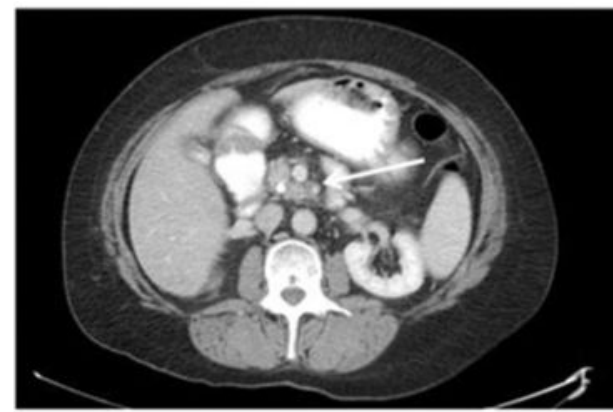

Post FOLFIRINOX

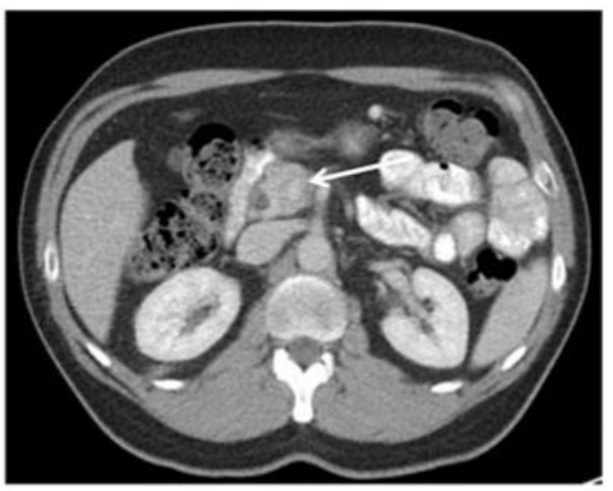

SMV involvement

Figure 2.

A, A 54-year-old woman with LA PDAC treated with 8 cycles FOLFIRINOX and 50.4 Gy of chemoradiation. Final pathology revealed a T3N0M0 PDAC with negative margin (R0). The patient is alive and free of disease at 25 months from diagnosis. B, 56-year-old man with LA PDAC treated with 6 cycles FOLFIRINOX and CyberKnife. Both images are of postneoadjuvant treatment. Final pathology revealed no viable tumor and negative lymph nodes. The patient is free of disease at 16 months from diagnosis. 


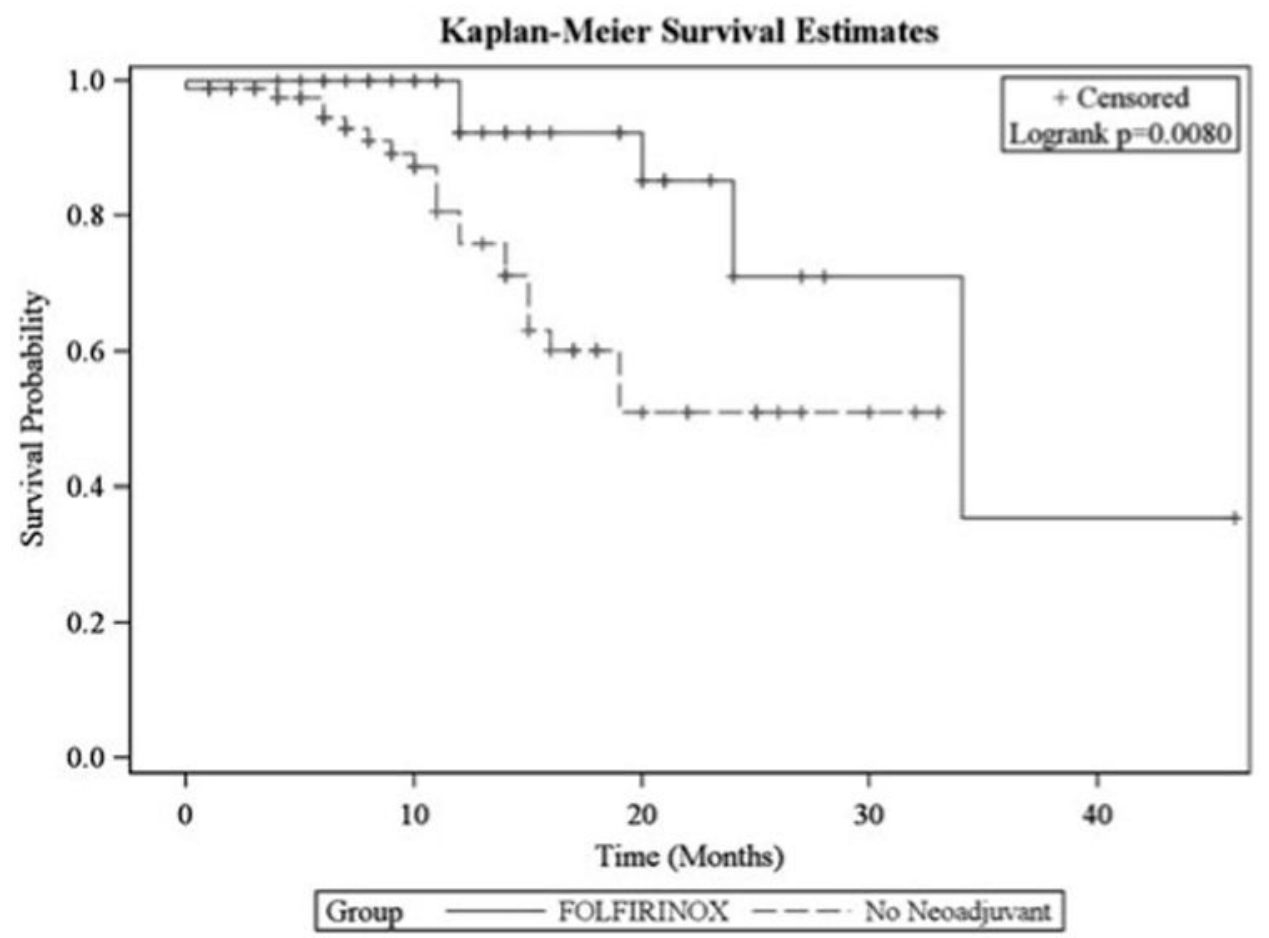

Figure 3.

Overall survival curves for patients who were treated with neoadjuvant FOLFIRINOX versus no neoadjuvant chemotherapy. 
Table 1

Demographics and Clinical Features of Study Population

\begin{tabular}{lccc}
\hline & No Neoadjuvant (N = 87) & FOLFIRINOX (N = 40) & $\boldsymbol{P}$ \\
\hline Male & $45(51.7 \%)$ & $21(52.5 \%)$ & 0.999 \\
Age, median (range), yr & $70(44-88)$ & $62(38-77)$ & $<0.001$ \\
Median Ca19-9 & $140(0-20,000)$ & $169(1-4754)$ & \\
Charlson comorbidity score, median (range) & $3(1-11)$ & $2(0-5)$ & $<0.001$ \\
& $0=44(50.6 \%)$ & $0=30(75 \%)$ & 0.013 \\
ECOG performance status & $1=34(39.1 \%)$ & $1=10(25 \%)$ & \\
& $2=9(10.3 \%)$ & $2=0$ & \\
BMI, kg/m ${ }^{2}$ & $27.3(18.5-50.1)$ & $24.5(19.1-43.2)$ & 0.038 \\
Weight loss $>$ 5 kg & $32(36.8 \%)$ & $19(47.5 \%)$ & 0.330 \\
Complete cycles FOLFIRINOX, median (range) & 0 & $8(1-24)$ & \\
\hline
\end{tabular}


Table 2

FOLFIRINOX Treated Patients

\begin{tabular}{|c|c|c|c|}
\hline & $\begin{array}{c}\text { Pre-FOLFIRINOX Treatment }(\mathrm{n} \\
=40)\end{array}$ & $\begin{array}{l}\text { Post-FOLFIRINOX Treatment } \\
\qquad(\mathrm{n}=40)\end{array}$ & $P$ \\
\hline CA 19.9, median (range) ${ }^{1}$ & $169(1-4754)$ & $0.17(0.01-9.81)$ & $<0.001$ \\
\hline CA $19.9>40 \mathrm{U}^{1}$ & $26(70.3 \%)$ & $11(28.9 \%)$ & $<0.001$ \\
\hline Tumor diameter at $\mathrm{CT}$, median (range), $\mathrm{cm}$ & $3.6(0-6.0)$ & $2.1(0-5.4)$ & $<0.001$ \\
\hline \multirow{4}{*}{ Gastrointestinal consensus group } & $\mathrm{LAPC}=25(62.5 \%)$ & Complete $=6(15 \%)$ & \\
\hline & & Partial $=30(75 \%)$ & \\
\hline & Borderline $=15(37.5 \%)$ & Stable $=4(10 \%)$ & \\
\hline & & Progression $=0$ & \\
\hline \multirow{3}{*}{ Blinded review by senior pancreatic surgeon (A.L.W.) } & Resectable -0 & Resectable-12 (30\%) & \\
\hline & Borderline-14 (35\%) & Borderline $-9(22 \%)$ & \\
\hline & LA $-26(65 \%)$ & LA-19 $(48 \%)$ & \\
\hline
\end{tabular}

LAPC indicates locally advanced pancreatic cancer. 
Table 3

Operative Outcomes

\begin{tabular}{|c|c|c|c|}
\hline & No Neoadjuvant ( $N=87$ ) & FOLFIRINOX $(\mathrm{N}=40)$ & $\boldsymbol{P}$ \\
\hline Operation & & & 0.276 \\
\hline Whipple & $63(73 \%)$ & $34(85 \%)$ & \\
\hline Distal pancreatectomy & $23(26 \%)$ & $6(15 \%)$ & \\
\hline Total pancreatectomy & $1(1 \%)$ & 0 & \\
\hline OR time (min) & $300(60-600)$ & $393.5(180-890)$ & $<0.001$ \\
\hline Blood loss (mL) & $400(100-1800)$ & $600(200-7800)$ & 0.007 \\
\hline Postoperative complications & $55(63 \%)$ & $14(36 \%)$ & 0.006 \\
\hline Pancreatic Fistula & $19(22 \%)$ & 0 & $<0.001$ \\
\hline LOS (median) & $7(4-54)$ & $6(4-35)$ & 0.145 \\
\hline Readmission (90 d) & $26(30 \%)$ & $8(20 \%)$ & 0.286 \\
\hline Postoperative death (within $90 \mathrm{~d}$ ) & $1(1.1 \%)$ & 0 & 0.999 \\
\hline Median FU (from diagnosis) & $10(0-33)$ & $13.5(4-46)$ & 0.003 \\
\hline Progression & $43(49.4 \%)$ & $15(37.5 \%)$ & 0.252 \\
\hline Progression pattern & $(\mathrm{N}=40)$ & $(\mathrm{N}=15)$ & 0.481 \\
\hline \multicolumn{4}{|l|}{ Local } \\
\hline Pancreas bed & $7(17.5 \%)$ & $4(26.7 \%)$ & \\
\hline Regional nodes & $4(10 \%)$ & 0 & \\
\hline \multicolumn{4}{|l|}{ Distant } \\
\hline Multiple metastases & $3(7.5 \%)$ & $3(20 \%)$ & \\
\hline Lungs & $4(10 \%)$ & $1(6.7 \%)$ & \\
\hline Liver & $17(42.5 \%)$ & $4(26.7 \%)$ & \\
\hline Peritoneum & $5(12.5 \%)$ & $3(20 \%)$ & \\
\hline Dead of disease & $21(24 \%)$ & $5(12.5 \%)$ & 0.160 \\
\hline
\end{tabular}


Table 4

Pathological Features of Study Population

\begin{tabular}{lrrc}
\hline & No Neoadjuvant $(\mathbf{N}=\mathbf{8 7})$ & FOLFIRINOX (N = 40) & \multicolumn{1}{c}{$\boldsymbol{P}$} \\
\hline Stage & & & $<0.001$ \\
$\quad$ I & $4(5 \%)$ & $10(25 \%)$ & 0.006 \\
IIa & $15(17 \%)$ & $16(40 \%)$ & \\
IIb & $68(78 \%)$ & $14(35 \%)$ & \\
Median size of tumor on pathology (cm) & $3.2(1.5-10.7)$ & $2.5(0.1-5.5)$ & \\
N+ & $69(79 \%)$ & $14(35 \%)$ & $<0.001$ \\
R0 & $75(86 \%)$ & $35(92 \%)$ & 0.550 \\
Lymphatic invasion & $61(70 \%)$ & $14(35 \%)$ & $<0.001$ \\
Perineural invasion & $83(95.4 \%)$ & $29(72.5 \%)$ & $<0.001$ \\
\hline
\end{tabular}

